\title{
Limited Add-On Effects of Unilateral and Bilateral Transcranial Direct Current Stimulation on Visuo-Motor Grip Force Tracking Task Training Outcome in Chronic Stroke. A Randomized Controlled Trial
}

OPEN ACCESS

Edited by:

Bernhard Elsner,

SRH Hochschule für

Gesundheit, Germany

Reviewed by:

Lukas J. Volz

University of Cologne, Germany Domenico Antonio Restivo,

Garibaldi Hospital, Italy

*Correspondence:

Agnes Flöe

agnes.floee/@med.uni-greifswald.de

tThese authors have contributed equally to this work

Specialty section:

This article was submitted to

Neurorehabilitation,

a section of the journal

Frontiers in Neurology

Received: 06 July 2021

Accepted: 01 October 2021

Published: 11 November 2021

Citation:

Taud B, Lindenberg R, Darkow $R$,

Wevers J, Höfflin D, Grittner U, Meinzer M and Flöel A (2021) Limited

Add-On Effects of Unilateral and

Bilateral Transcranial Direct Current Stimulation on Visuo-Motor Grip Force

Tracking Task Training Outcome in

Chronic Stroke. A Randomized

Controlled Trial.

Front. Neurol. 12:736075.

doi: 10.3389/fneur.2021.736075

\section{Benedikt Taud ${ }^{1}$, Robert Lindenberg 1,2, Robert Darkow ${ }^{1}$, Jasmin Wevers ${ }^{1}$, Dorothee Höfflin ${ }^{1}$, Ulrike Grittner ${ }^{3,4}$, Marcus Meinzer ${ }^{1,5+}$ and Agnes Flöel ${ }^{1,5,6,7 * t}$}

\footnotetext{
'Neurocure Cluster of Excellence, Charité University Medicine, Berlin, Germany, ${ }^{2}$ Department of History, Philosophy and Ethics of Medicine, Heinrich Heine University, Düsseldorf, Germany, ${ }^{3}$ Berlin Institute of Health at Charité, Charité University Medicine, Berlin, Germany, ${ }^{4}$ Institute of Biometry and Clinical Epidemiology, Charité University Medicine, Berlin, Germany, ${ }^{5}$ Department of Neurology, University Medicine Greifswald, Greifswald, Germany, ${ }^{6}$ German Centre for Neurodegenerative Diseases, Site Greifswald/Rostock, Greifswald, Germany, ${ }^{7}$ Center for Stroke Research, Charité University Medicine, Berlin, Germany
}

Background: This randomized controlled trial investigated if uni- and bihemispheric transcranial direct current stimulation (tDCS) of the motor cortex can enhance the effects of visuo-motor grip force tracking task training and transfer to clinical assessments of upper extremity motor function.

Methods: In a randomized, double-blind, sham-controlled trial, 40 chronic stroke patients underwent 5 days of visuo-motor grip force tracking task training of the paretic hand with either unilateral or bilateral ( $N=15 /$ group) or placebo tDCS ( $N=10)$. Immediate and long-term (3 months) effects on training outcome and motor recovery (Upper Extremity Fugl-Meyer, UE-FM, Wolf Motor Function Test, and WMFT) were investigated.

Results: Trained task performance significantly improved independently of tDCS in a curvilinear fashion. In the anodal stimulation group UE-FM scores were higher than in the sham group at day 5 (adjusted mean difference: 2.6, 95\%Cl: 0.6-4.5, $p=0.010$ ) and at 3 months follow up (adjusted mean difference: 2.8, 95\%Cl: 0.8-4.7, $p=0.006$ ). Neither training alone, nor the combination of training and tDCS improved WMFT performance.

Conclusions: Visuo-motor grip force tracking task training can facilitate recovery of upper extremity function. Only minimal add-on effects of anodal but not dual tDCS were observed.

Clinical Trial Registration: https://clinicaltrials.gov/ct2/results?recrs=\&cond=\&term= NCT01969097\&cntry=\&state=\&city=\&dist=, identifier: NCT01969097, retrospectively registered on 25/10/2013.

\footnotetext{
Keywords: chronic stroke, tDCS, motor function, motor rehabilitation, hand function
} 


\section{BACKGROUND}

Stroke is the leading cause of acquired disability worldwide (13 ). The most common consequence of a stroke is a hemiparesis of the contralesional upper limb. Approximately $80 \%$ of all stroke patients are initially affected and persistent (chronic) impairment of upper extremity functioning is observed in $\sim 40 \%$ of stroke patients, resulting in reduced quality of life, participation in societal activities, and lower odds for successful vocational reintegration (4). Numerous motor rehabilitation strategies, like physical or occupational therapy, have been investigated that aim at improving upper extremity function [for reviews see $(5,6)]$. However, treatment effects can be highly variable and frequently only small to moderate effect sizes and limited transfer to activities of daily living have been reported (7-10).

Consequently, development of adjunct approaches that aim at enhancing the effects of behavioral interventions, such as noninvasive brain stimulation, have recently received substantial attention. In particular, the combination of behavioral treatment with repetitive transcranial magnetic stimulation (rTMS) or transcranial direct current stimulation (tDCS) can have positive effects on motor rehabilitation after stroke $(11,12)$. During rTMS, a strong magnetic field is used to induce electrical activity in the underlying brain area. Depending on the frequency, intensity, and duration of the stimulation, rTMS is able to increase or decrease cortical excitability (13). Low frequency stimulation $(<1 \mathrm{~Hz})$ results in decreased cortical excitability, whereas high frequency stimulation $(<1 \mathrm{~Hz})$ results in increased cortical excitability (13-15). TDCS involves administration of a weak electrical current via scalp attached electrodes. Acute stimulation effects are mediated by modulation of the neural resting-membrane potential, resulting in changes in cortical excitability in underlying cortical regions. Depending on the polarity of the current, tDCS can be used to increase or decrease cortical excitability (16), with anodal tDCS yielding a relative depolarization and cathodal tDCS yielding hyperpolarization of neuronal membranes (17). Long-term effects of the stimulation are thought to depend on synaptic mechanisms mimicking longterm potentiation, which are critical for neural plasticity and learning (18). The current study utilized tDCS because it can be administered concurrently with motor training, while offering a superior safety profile and placebo stimulation condition compared to rTMS (19).

In motor rehabilitation after stroke, three different tDCS electrode set-ups have frequently been used and are based on different assumptions regarding neural recovery (20): (1) Unihemispheric excitatory ("anodal") tDCS targeting the ipsilesional motor cortex is used to facilitate neural function regions that are spared by the stroke. (2) Unihemispheric inhibitory ("cathodal") tDCS targeting the contralesional motor cortex is used to reduce activity in regions that may potentially interfere with motor behavior. (3) Bihemispheric "dual" tDCS combines both approaches and aims at facilitating ipsilesional neural activity, while inhibiting contralesional regions (21-25). Several studies have reported beneficial tDCS effects on motor rehabilitation using anodal (26-29), cathodal (30-32), or dual set-ups $(21,22,33)$. However, few studies have directly compared the effects of different set-ups $(31,34,35)$. Moreover, with regard to upper extremity functioning, substantial previous research has focused on training of whole arm movements $(21,22,36,37)$. However, severely impaired patients may not be able to execute extensor muscle movements which are required for these training paradigms [e.g., constraint-induced movement therapy, CIMT, (38)]. To address this problem, the effectiveness of treatment approaches targeting fine motor control of the paretic hand have been explored $(38,39)$. Indeed, many severely affected patients are still able to generate whole hand grip force $(40,41)$ and improvement of grip force is associated with improvements of upper limb functional status (42). Moreover, a previous study demonstrated improvement on the Upper-Extremity Fugl-Meyer (UE-FM) elbow scale through visuo-motor training. However, no additional gains were induced by anodal tDCS (38), possibly related to the low current strength $(0.5 \mathrm{~mA})$ that was used. In addition, it has not yet been investigated whether dual tDCS might also have a positive effect on visuo-motor grip force tracking task training.

Therefore, the present study aimed to address three open questions: (1) Does visuo-motor grip force tracking task training improve performance in the trained task, and/or generalizes to clinical assessments of upper extremity function in chronic stroke? (2) If immediate and long-term training gains occur, are they enhanced by concurrent unilateral or bilateral motor cortex tDCS? (3) Does unilateral or bilateral tDCS result in differential transfer effects? We hypothesized that both anodal and dual tDCS would enhance motor training outcome and transfer to clinical assessments of upper motor function. Effects of contralesional cathodal tDCS alone were not examined because negative effects were reported in chronic patients with moderate or severe impairment (43).

\section{MATERIALS AND METHODS}

Forty patients with chronic ( $>6$ months post-stroke; see Table 1 for details) right or left hemispheric ischemic or hemorrhagic stroke participated in a randomized, double-blind, shamcontrolled study. Inclusion criteria were the occurrence of an ischemic or hemorrhagic stroke at least 6 month prior to enrollment; no subsequent strokes; no additional neurological, medical, or psychiatric disorders or contraindication for tDCS (e.g., skull fractures or metal implants); no concurrent use of CNS-affecting drugs and the ability to complete the motor training.

Eligible patients were stratified to the stimulation groups by baseline UE-FM score to receive 5 consecutive days of visuo-motor grip force tracking task training with either anodal $(N=15)$, dual $(N=15)$, or placebo ("sham"; $N=10)$ tDCS. Patients, care providers and investigators were blinded to the stimulation conditions. Sample size estimations were based on previous uni- and bihemispheric tDCS studies $(22,24)$. The study was approved by the local ethics committee. Participants gave written informed consent prior to study inclusion. The trial was registered (NCT01969097). Figure 1 displays the flow-chart of the study. 
TABLE 1 | Demographic information and baseline motor performance.

\begin{tabular}{|c|c|c|c|c|c|c|}
\hline Group & Age, years & $\begin{array}{l}\text { Time post-stroke, } \\
\text { months }\end{array}$ & $\operatorname{Sex}\left(0^{x} / \%\right)$ & $\begin{array}{l}\text { Baseline } \\
\text { UE-FM }\end{array}$ & $\begin{array}{l}\text { Baseline } \\
\text { WMFT }\end{array}$ & $\begin{array}{c}\text { Affected hemisphere } \\
\text { (right/left) }\end{array}$ \\
\hline Dual & $58.3 \pm 12.8$ & $21.9 \pm 17.2$ & $11 / 4$ & $47.1 \pm 17.9$ & $0.7 \pm 0.6$ & $8 / 7$ \\
\hline Anodal & $60.3 \pm 10.3$ & $28.8 \pm 35.3$ & $12 / 3$ & $46.9 \pm 15.0$ & $0.6 \pm 0.6$ & $8 / 7$ \\
\hline Sham & $60.6 \pm 12.9$ & $28 \pm 25.1$ & $8 / 2$ & $43.6 \pm 20.7$ & $0.8 \pm 0.7$ & $6 / 4$ \\
\hline Mean SMD & 0.13 & 0.19 & 0.11 & 0.13 & 0.17 & 0.09 \\
\hline
\end{tabular}

Values are reported as mean \pm standard deviation, SMD, standardized mean difference.

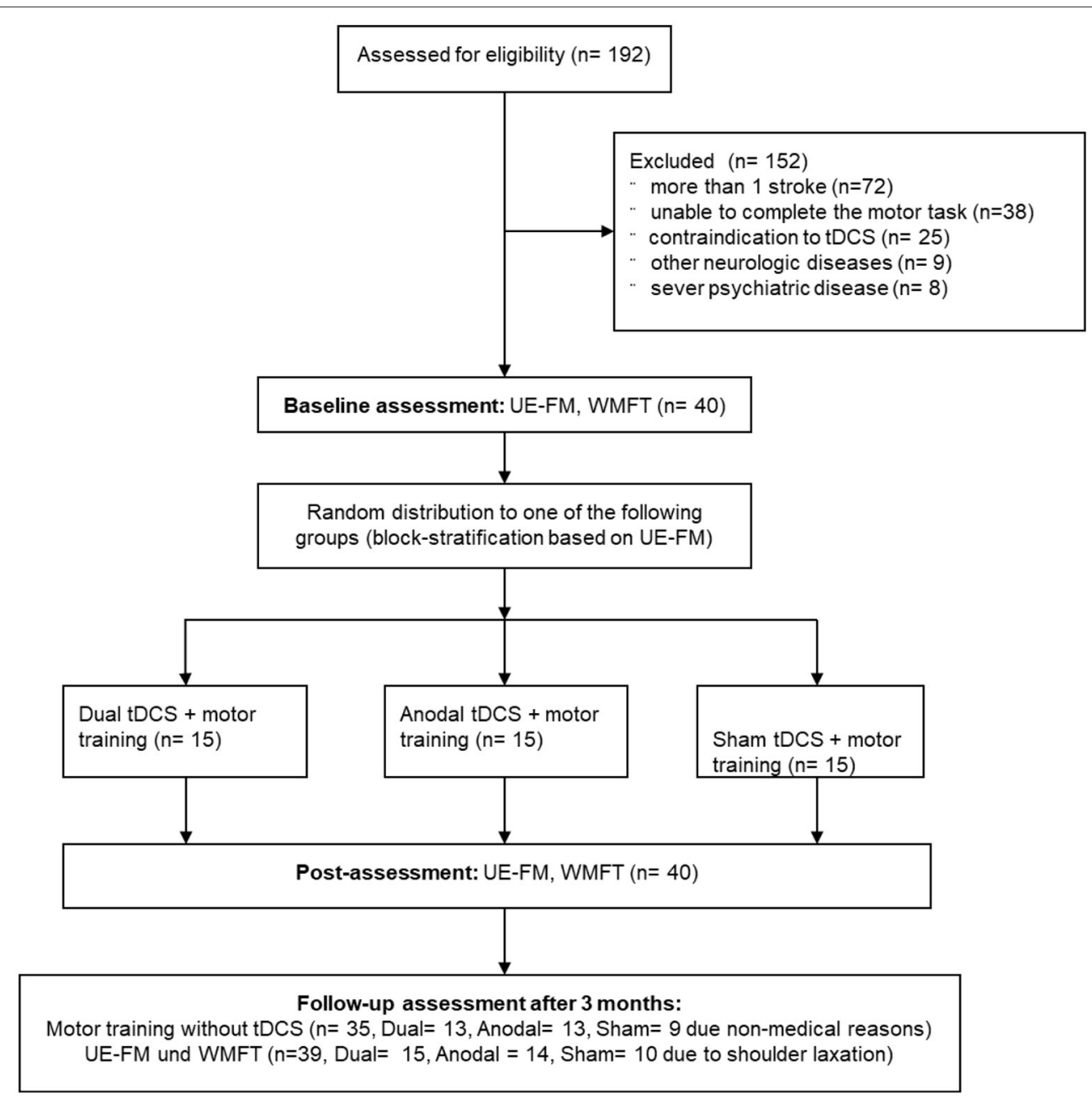

FIGURE 1 | Study flow chart. UE-FM, Upper Extremity Fugl-Meyer; WMFT, Wolf Motor Function Test; tDCS, transcranial direct current stimulation.

Primary and Secondary Research Question

The primary research question was whether unilateral or bilateral tDCS in combination with a visuo-motor grip force tracking task training improves UE-FM scores in stroke patients. Changes in training performance and WMFT scores were secondary outcomes.

\section{Clinical Assessment}

All patients underwent standardized assessments of motor function and impairment using UE-FM and WMFT that were administered prior to and immediately after the intervention.
The UE-FM examines multi-joint movements of the upper limb $[$ max. score $=66$, lower scores $=$ greater impairment; (44)] . The WMFT comprises 15 time-based items ranging from whole arm movements to fine finger control. WMFT completion times were logarithmized to account for skewed data distribution (45). This score has a maximum value of $2.08 \mathrm{~s}[\log ]$ with lower values reflecting better arm function. All tests (including the training task without-tDCS) were repeated 3 months later to investigate potential long-term effects of tDCS on motor function. Assessments were videotaped and analyzed by two independent raters. 

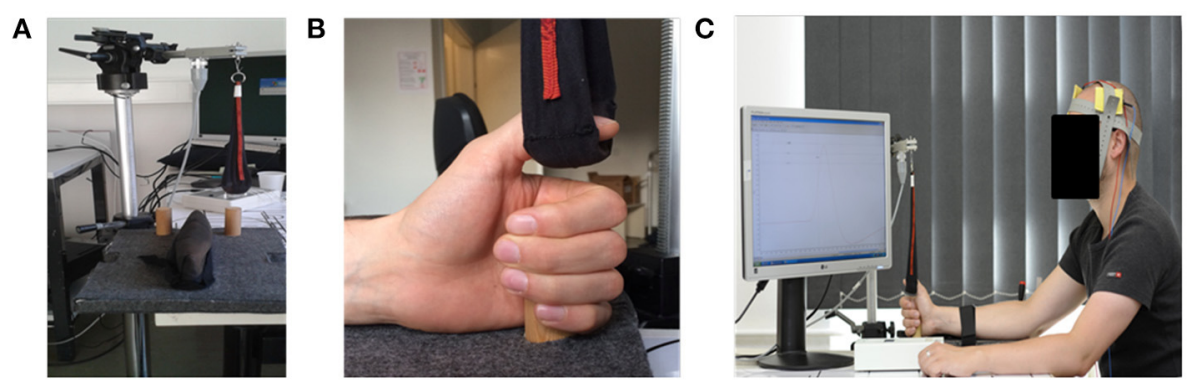

FIGURE 2 | Experimental set-up. (A) The Grass ${ }^{\circledR}$ Force Displacement Transducer FT10, the sling to measure the applied strength and the grip board. (B) Shows a left hand grasping the wooden grip while the thumb is placed in the sling connected to the Force Displacement Transducer. (C) Illustrates the overall set-up with the dual tDCS montage.

One participant (anodal-group) was excluded from UEFM/WMFT follow-up assessments due to a shoulder subluxation. Follow-up motor task data of five participants ( 2 dual, 2 anodal, 1 sham) was not recorded correctly due to technical difficulties and could not be used in the analysis.

\section{Motor Training}

Details of the visuo-motor grip force tracking task have been described previously (46). In short, patients performed isometric adductions with their paretic thumb. They sat comfortably in front of a computer screen and were asked to hold on to a wooden grip protruding vertically from the table with their paretic hand. Their thumb was placed in a sling attached to a Grass ${ }^{\circledR}$ Force Displacement Transducer FT10 (Grass Instruments). Velcro straps were used to fixate the forearm in order to minimize unwanted movement. The set-up is displayed in Figure 2. Signal software (Cambridge Electronic Design Ltd.) was used for data acquisition and task presentation. Force displacement was amplified and digitized using a CPT22 AC/DC Straining Gage Amplifier (Grass ${ }^{\circledR}$ Technologies) with an amplification of $2,000 \mathrm{~Hz}$ and a high filter of $3 \mathrm{~Hz}$.

At the beginning of each session, the patients abducted their thumb five times as hard as possible in order to establish a maximum force output for each individual session. A target force window was then defined as the range between 30 and $40 \%$ of the individual maximum force output and displayed as three horizontal lines $(30,35$, and $40 \%)$ on the computer screen. Each experimental display was scaled individually based on the patient's individual maximum force output. A horizontal line representing $40 \%$ was located $9 \mathrm{~cm}$ from the top of the screen, and a line representing $30 \%$ was located $13 \mathrm{~cm}$ from the top of the screen. In between, a line representing 35\% was displayed. The zero force line was positioned at the bottom of the screen.

Each trial lasted $4 \mathrm{~s}$ and at the $3 \mathrm{~s}$ mark a vertical line was displayed as reference for the participants. During each trial, a red line moved in real time across the screen from left to right at zero force. Whenever pressure was applied via the force transducer, the line moved upwards accordingly and returned to zero force after the pressure was released. Participants were asked to abruptly apply pressure via their thumb in a manner that caused the red line to reach its highest point as close as possible to the intersection of the $35 \%$ line and a vertical line displayed at the $3 \mathrm{~s}$ mark, before returning to zero force. A trial was scored as "hit" if participants managed to place the red line's maximum between the $30 \%$ (bottom) and $40 \%$ (top) lines. All other trials were scored as "miss." A typical trial-run is displayed in Figure 3. Since participants were required to apply only $30-40 \%$ of their maximum force output, this was considered a visuo-motor grip force tracking task training.

During each motor training session, participants completed 240 trials across eight blocks of 30 trials each. In between blocks they were given a $60 \mathrm{~s}$ rest period, resulting in a total training time of $\sim 23 \mathrm{~min}$.

\section{Direct Current Stimulation}

A DC-Stimulator PLUS (NeuroConn, Germany) was used for stimulation with the active anode $\left(5 \times 7 \mathrm{~cm}^{2}\right)$ placed over the ipsilesional primary motor cortex according to the international 10-20 EEG system (i.e., C3 or C4, depending on site of the lesioned hemisphere). In the unilateral (anodal tDCS only) condition, the cathode $\left(10 \times 10 \mathrm{~cm}^{2}\right)$ was placed over the contralesional supraorbital ridge. The large size of the cathode rendered the stimulation functionally inert during this condition. In the dual condition, a smaller active cathode (5 $\times 7 \mathrm{~cm}^{2}$ ) was placed over the contralesional primary motor cortex. In the sham condition, the electrode set-up was pseudorandomly assigned to participants (either anodal or dual) and balanced across the group. Direct current was increased to $1 \mathrm{~mA}$ over $10 \mathrm{~s}$ and lasted for $23 \mathrm{~min}$ in the anodal and dual conditions. In the sham condition, it was ramped down after $30 \mathrm{~s}$. This procedure has resulted in successful participant blinding in previous studies [see (47)]. The tDCS parameters were within established safety guidelines (48). A second investigator configured the DC-Stimulator PLUS in order to ensure investigator blinding.

\section{Statistical Analysis}

SPSS 22 (IBM Corp. Released 2013) and R [packages: lme4, r2glmm, tidyverse, ggeffects (49-53)] and a two-sided 


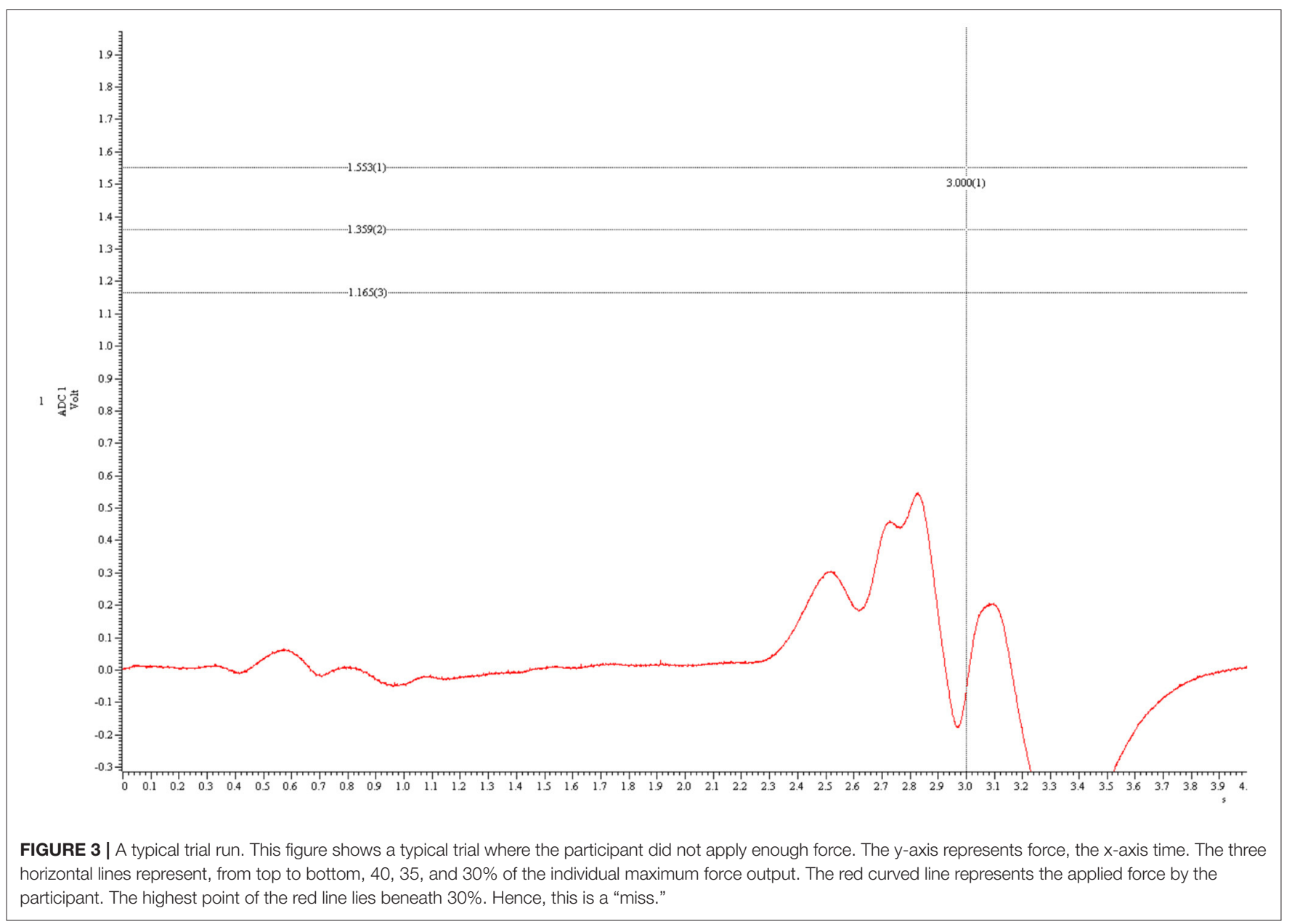

significance level (alpha $=0.05$ ) was employed. Separate linear mixed models (54) investigated effects of the active stimulation conditions compared to sham tDCS on performance (motor task, UE-FM, WMFT). Time points (motor task: training-days $1-5$, follow-up; UE-FM/WMFT: baseline, post, follow-up) were level-one units nested in different individuals (level-two units). Random intercept models tested differences between the stimulation conditions. A squared centered time variable $\left(\mathrm{TIME}^{2}\right)$ tested for curvilinear learning effects in the regression model for motor task. The Time $\times$ Stimulation interaction assessed whether the slopes differed between groups. Baseline UE-FM scores and training blocks were covariates in the motor task analysis. For models testing stimulation effects on UE-FM and WMFT, the respective baseline values were included as covariates as well as the time point of measurement (day 5, 3 month follow up) and the interaction of stimulation group and time point. In order to investigate effects of active stimulation, we also conducted linear mixed models testing the combined stimulation effect of anodal and dual stimulation vs. sham tDCS (this analysis was reported in the Supplementary Material along with training outcomes for individual patients, see Supplementary Figures 1-3). There was no adjustment for multiple testing and $p$-values. See Supplementary Materials for full model estimates.

\section{RESULTS}

\section{UE-FM}

Adjusted UE-FM scores at day 5 were 47.0 (95\%CI: 45.5-48.5) for sham, 48.1 (95\%CI: 46.9-49.3, difference to sham: 1.1, 95\%CI: -0.8 to $3.0, p=0.251$ ) for dual, and 49.6 (95\% CI: $48.3-$ 50.8, difference to sham: $2.6,95 \% \mathrm{CI}: 0.6-4.5, p=0.010)$ for anodal (Figure 4). Three month later the adjusted UE-FM scores were: 47.4 (95\%CI: 45.9-48.9) for sham, 48.7 (95\%CI: 47.5-49.9, difference to sham: $1.3,95 \% \mathrm{CI}:-0.6$ to $3.2, p=0.177)$ for dual, and 50.2 (95\%CI: 48.9-51.4, difference to sham: 2.8, 95\%CI: 0.8$4.7, p=0.006)$ for anodal. In the anodal stimulation group the values were highest. However, differences between anodal and dual were small at day 5 (mean difference: $1.5,95 \% \mathrm{CI}:-0.3$ to $3.2, p=0.094$ ) and at 3 months follow up (mean difference: 1.5 , $95 \% \mathrm{CI}:-0.3$ to $3.2, p=0.095)$.

\section{Motor Training}

There was no overall difference between the groups with regard to hits (reference sham: dual $\beta=-0.73,95 \% \mathrm{CI}$ : -3.76 to 


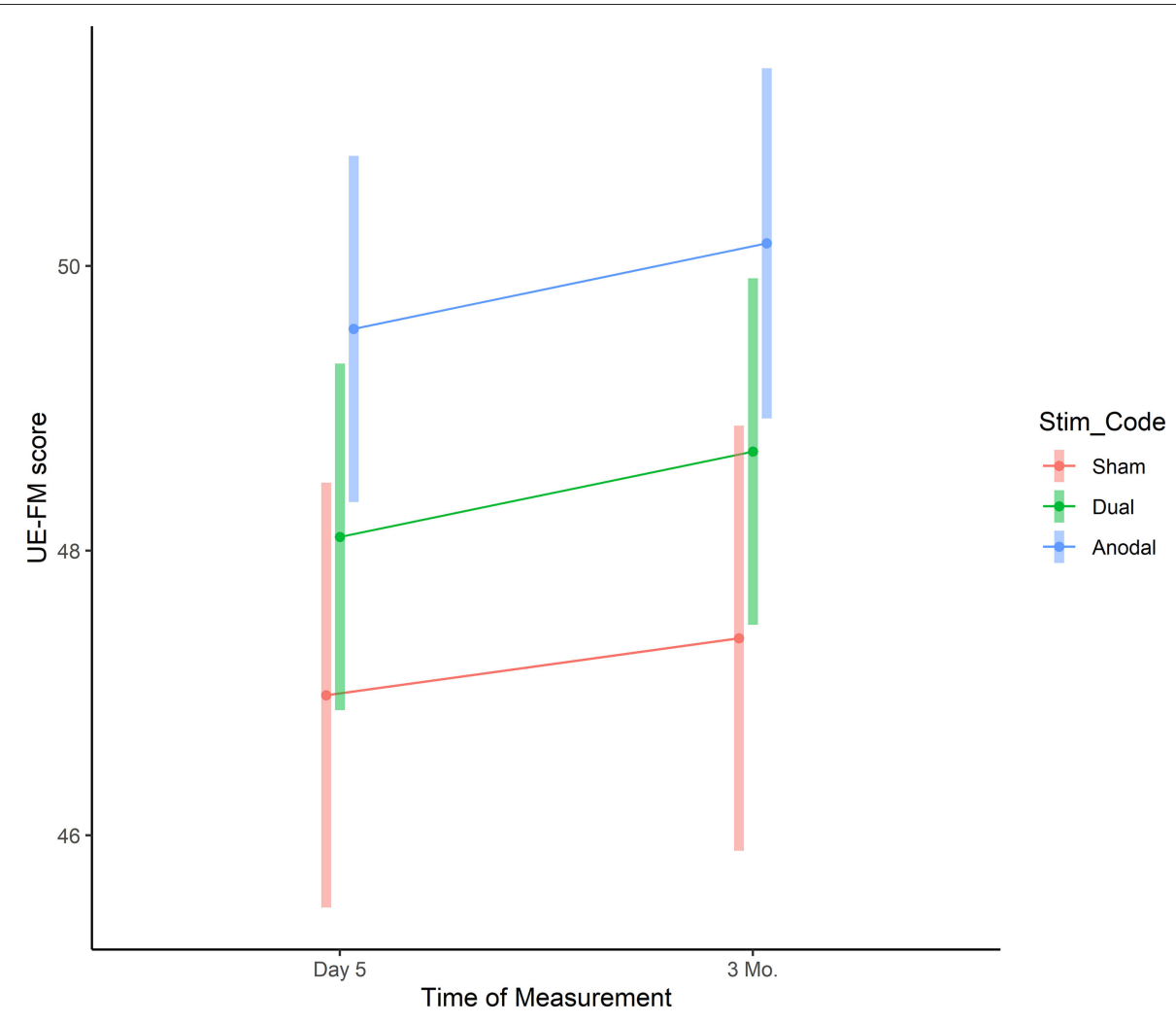

FIGURE 4 | UE-FM assessment. The graphs depict the adjusted mean UE-FM score for each group at post-assessment and follow-up period based on the linear mixed model. Vertical bars represent model based 95\% confidence intervals.

2.31, $p=0.64, R^{2}=0.01$; anodal $\beta=0.88,95 \% \mathrm{CI}:-2.16$ to $\left.3.91, p=0.58, R^{2}=0.01\right)$. There was a curvilinear improvement in performance (TIME $\beta=-0.28,95 \% \mathrm{CI}:-0.34$ to $-0.21, p<0.001, R^{2}=0.03$, TIME $\beta=0.50$, $95 \% \mathrm{CI}$ : 0.31-0.70, $p<0.001, R^{2}=0.01$ ), and this effect tended to be more pronounced over time in both active stimulation groups compared to sham (dual $\times$ time, $\beta=0.17,95 \% \mathrm{CI}:-0.08$ to $0.43, p=0.18, R^{2}=0.00$; anodal $\times$ time, $\beta=0.25$, 95\%CI: -0.007 to $0.50, p=0.06, R^{2}=0.00$; Figure 5). Model based estimates at day 5 were 17.2 (95\%CI: 14.7-19.6) for sham, 16.8 (95\%CI: 14.8-18.8) for dual, and 18.5 (95\%CI: 16.5-20.5) for anodal stimulation (pairwise comparisons: all $p>0.32$ ).

\section{WMFT}

Adjusted WMFT scores at day 5 were 0.66 (95\%CI: $0.60-$ 0.72 ) for sham, 0.62 (95\%CI: 0.57-0.67, difference to sham: $-0.04,95 \% \mathrm{CI}$ : -0.11 to $0.04, p=0.360$ ) for dual, and 0.63 (95\%CI: $0.59-0.68$, difference to sham: $-0.02,95 \% \mathrm{CI}:-0.10$ to $0.05, p=0.518$ ) for anodal (Figure 6). Three month later the adjusted WMFT scores were: 0.66 (95\%CI: $0.60-0.72)$ for sham, 0.62 (95\%CI: 0.57-0.67, difference to sham: $-0.04,95 \% \mathrm{CI}$ : -0.11 to $0.04, p=0.323$ ) for dual, and 0.60 (95\%CI: $0.55-0.65$, difference to sham: $-0.05,95 \% \mathrm{CI}$ : -0.13 to $0.02, p=0.161$ ) for anodal.

\section{DISCUSSION}

The results of this randomized, controlled clinical trial demonstrated that a visuo-motor grip force tracking task training, consisting of isometric abductions of the paretic thumb, improved control of the paretic thumb, and resulted in generalization to clinical assessments of upper extremity function such as UE-FM, but not WMFT, in chronic stroke patients. Compared to sham tDCS, UE-FM performance improvement was more pronounced in the group that had received unilateral (anodal) tDCS. However, this effect was relatively small (Cohen's $d=0.34$ ) and those add-on effects were not significantly different from bilateral (dual) tDCS. No improvement on the WMFT was found.

Previous studies have reported that 4 weeks using comparable isometric pinch task resulted in better trained task performance and improvements of UE-FM, but not WMFT (38). Similarly, in a sample of well-recovered stroke patients, 1 week of training on a sequential visual isometric pinch force task resulted in better task performance, improvements in the Jebsen Taylor hand function test (JTT) and the Grooved Pegboard Test (GPT) (55). Our study is in line with these findings by showing that visuo-motor grip force tracking task training can result in long-term improvements of trained task performance and functionally relevant recovery of upper extremity function. 


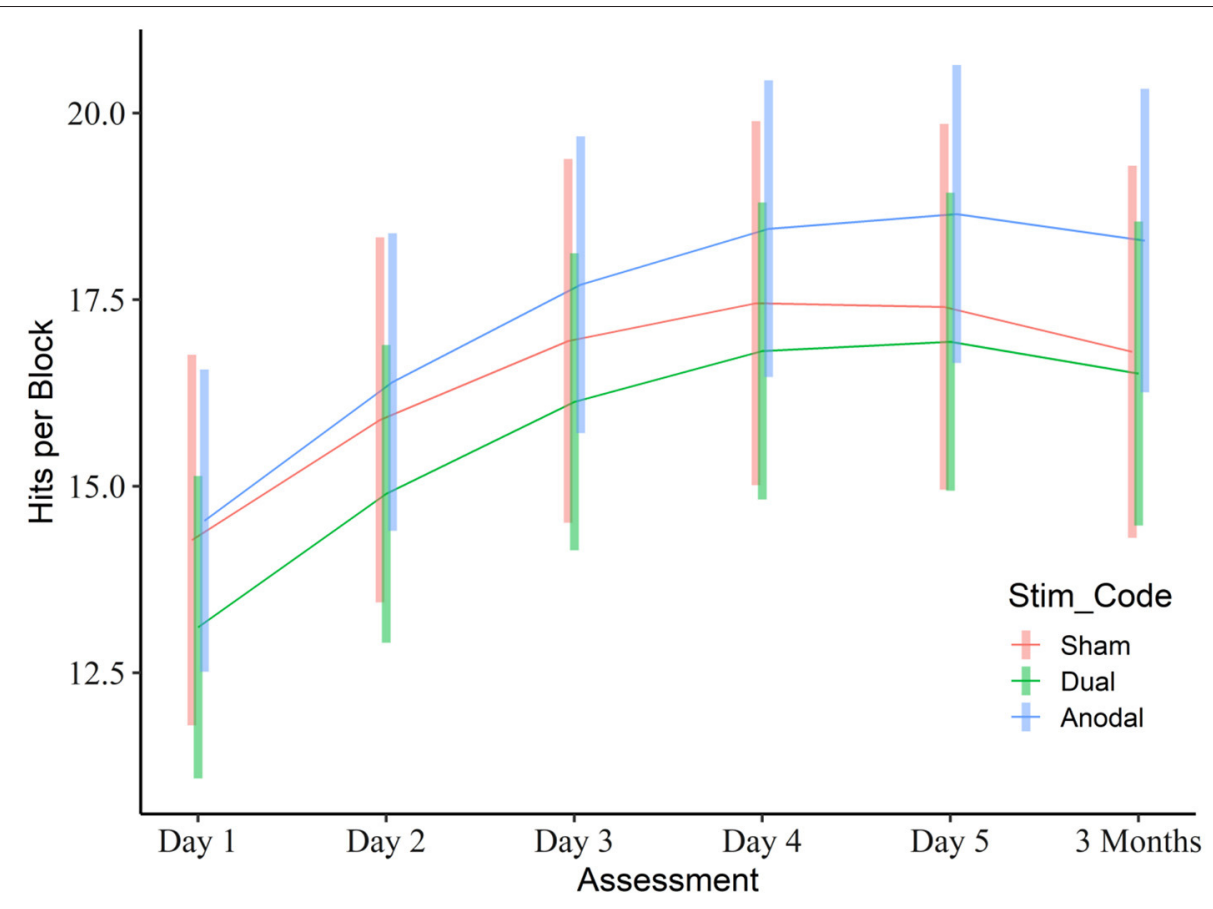

FIGURE 5 | Learning curve in the visuo-motor grip force tracking task training task. The graph depicts the mean number of hits per block for each group across the training and follow-up period based on the linear mixed model. Vertical bars represent model based $95 \%$ confidence intervals.

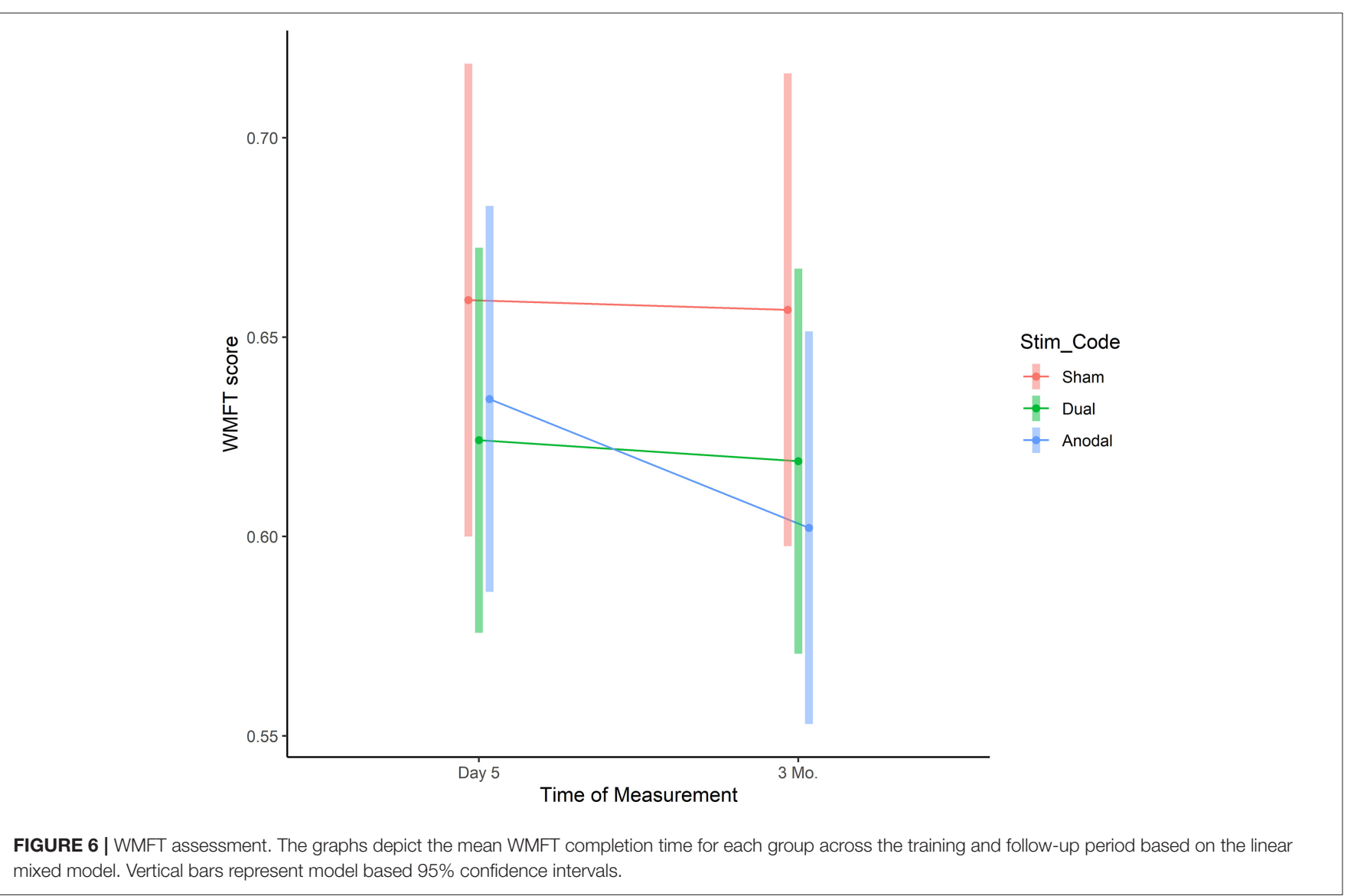


However, the present study did not confirm substantial add-on effects of unilateral or bilateral tDCS. While anodal tDCS selectively resulted in steeper gains on the UE-FU, these effects were small and not statistically different from bilateral stimulation. No stimulation effects were found on trained task performance or the WMFT.

Previous studies had only investigated effects of unilateral tDCS on visuo-motor grip force tracking task training and reported mixed results. While, Pavlova et al. (38) demonstrated beneficial effects of anodal tDCS on the shoulder-elbow subscale of the UE-FM, no effects on trained task performance were found. In contrast, Hamoudi et al. (55) reported improved trained task performance with anodal tDCS, but not on the JTT and GPT. Both studies reported no long-term effects of anodal tDCS on task performance, but beneficial effects on the shoulderelbow subscale were maintained for up to 2 months (38). Therefore, mainly weak effects of anodal tDCS on visuo-motor grip force tracking task training have been reported previously. Together with the results of our own study, this questions the utility of tDCS to enhance the outcome of this particular type of training. Nonetheless, while there were no substantial effects at the group level, individual participants may have benefited from $\mathrm{tDCS}$ and future studies are need to include individualized modeling of current flow and functional imaging to investigate characteristics of potential responders.

\section{Limitations}

The results of this study should be interpreted with caution due to the small sample size and high inter-individual variance in baseline motor function, lesion site, location and extent, time since stroke, age, and gender and missing data at the follow-up assessment (5/40 patients) and these factors may have contributed to the lack of effects in this study. Nonetheless, the missing data was balanced across the stimulation conditions and the mixed effects models used are robust regarding missing data. Furthermore, we also conducted a power analysis based on the strongest effect (i.e., the comparison of UE-FM scores between anodal vs. sham tDCS at day 5 and the 3 months follow up). At day 5, raw mean and SD values for anodal UE-FM were 50.4 (SD: 14.3 ), and for sham 44.6 (SD: 20.9) resulting in a standardized effect size (Cohen's d) of 0.34 . To demonstrate effects of 0.34 or larger using a two sample $t$-test and a two-sided significance level of $\alpha=0.05$ and a power of $0.8,139$ individuals per group would have to be included for a significant effect in a new study. Please note, with the current sample size $(N=40)$, we achieved an effect size of 0.261 between the three groups (ratio of variance of the means by the within group variance; significance level of 0.05 ,

\section{REFERENCES}

1. Global Burden of Disease. Global, regional, and national life expectancy, allcause mortality, and cause-specific mortality for 249 causes of death, 19802015: a systematic analysis for the Global Burden of Disease Study 2015. Lancet. (2016) 388:1459-544. doi: 10.1016/S0140-6736(16)31012-1

2. Hennerici MG, Kern R. S1-Leitlinie Diagnostik akuter zerebrovaskulärer Erkrankungen. 2017. In: Deutsche Gesellschaft für Neurologie, Hrsg. Leitlinien power $80 \%$ in a one-way analysis of variance). This highlights that smaller proof-of-principle studies are imperative prior to investing limited resources into larger randomized controlled trials and our results in combination with previous studies do not encourage such follow-up trials for the combination of tDCS and visuo-motor grip force tracking task training.

\section{Conclusions and Outlook}

Our results demonstrated significant performance improvements due to the visuo-motor grip force tracking task training in chronic patients with motor stroke. However, only limited addon effects were induced by unilateral anodal tDCS. Dual tDCS did not improve training outcome.

\section{DATA AVAILABILITY STATEMENT}

The raw data supporting the conclusions of this article will be made available upon reasonable request.

\section{ETHICS STATEMENT}

The studies involving human participants were reviewed and approved by Ethics Committee of the Charité Universitätsmedizin Berlin (Protocol: EA1/026/11). The patients/participants provided their written informed consent to participate in this study.

\section{AUTHOR CONTRIBUTIONS}

AF and RL conceived the study. BT, RD, DH, and JW processed the data. BT and UG performed the statistical analysis. BT wrote the manuscript. AF and MM revised the manuscript. All authors contributed to the article and approved the submitted version.

\section{FUNDING}

This work was supported by the Else Kröner-Fresenius Stiftung (RL:2011-119), Deutsche Forschungsgemeinschaft (AF:DFG-Exc-257; SFB 1315, Project \#327654276), and Bundesministerium für Bildung und Forschung (AF and MM:01EO0801).

\section{SUPPLEMENTARY MATERIAL}

The Supplementary Material for this article can be found online at: https://www.frontiersin.org/articles/10.3389/fneur. 2021.736075/full\#supplementary-material 
Stroke. (2002) 33:2718-21. doi: 10.1161/01.str.0000035286.87 503.31

5. Cramer SC. Repairing the human brain after stroke : I. Mechanisms of spontaneous recovery. Ann Neurol. (2008) 63:272-87. doi: 10.1002/ana.21393

6. Hatem SM, Saussez G, della Faille M, Prist V, Zhang X, Dispa D, Bleyenheuft Y. Rehabilitation of motor function after stroke: a multiple systematic review focused on techniques to stimulate upper extremity recovery. Front Hum Neurosci. (2016) 10:442. doi: 10.3389/fnhum.2016.00442

7. Pollock A, Farmer SE, Brady MC, Langhorne P, Mead GE, Mehrholz J, et al. Interventions for improving upper limb function after stroke. Cochrane Database Syst Rev. (2014) 2014:CD010820. doi: 10.1002/14651858.CD010820

8. Prabhakaran S, Zarahn E, Riley C, Speizer A, Chong JY, Lazar RM, et al. Interindividual variability in the capacity for motor recovery after ischemic stroke. Neurorehabil Neural Repair. (2008) 22:64-71. doi: 10.1177/1545968307305302

9. van der Vliet R, Selles RW, Andrinopoulou ER, Nijland R, Ribbers GM, Frens MA, et al. Predicting upper limb motor impairment recovery after stroke: a mixture model. Ann Neurol. (2020) 87:383-93. doi: 10.1002/ana.25679

10. Winters C, Van Wegen EEH, Daffertshofer A, Kwakkel G. Generalizability of the proportional recovery model for the upper extremity after an ischemic stroke. Neurorehabil Neural Repair. (2015) 29:614-22. doi: 10.1177/1545968314562115

11. Bai X, Guo Z, He L, Ren L, McClure MA, Mu Q. Different therapeutic effects of transcranial direct current stimulation on upper and lower limb recovery of stroke patients with motor dysfunction: a meta-analysis. Neural Plast. (2019) 2019:1372138. doi: 10.1155/2019/1372138

12. Hoyer EH, Celnik PA. Understanding and enhancing motor recovery after stroke using transcranial magnetic stimulation. Restor Neurol Neurosci. (2011) 29:395-409. doi: 10.3233/RNN-2011-0611.Understanding

13. Maeda F, Keenan JP, Tormos JM. Interindividual variability of the modulatory effects of repetitive transcranial magnetic stimulation on cortical excitability. Exp Brain Res. (2000). 425-30. doi: 10.1007/s002210000432

14. Hess G, Donoghue JP. Long-term depression of horizontal connections in rat motor cortex. Eur J Neurosci. (2006) 8:658-65. doi: 10.1111/j.1460-9568.1996.tb01251.x

15. Wang $\mathrm{H}$, Wang $\mathrm{X}$, Scheich $\mathrm{H}$. LTD and LTP induced by transcranial magnetic stimulation in auditory cortex. Neuroreport. (1996) 7:521-5.

16. Nitsche MA, Paulus W. Sustained excitability elevations induced by transcranial DC motor cortex stimulation in humans. Neurology. (2001) 57:39-40. doi: 10.1212/WNL.57.10.1899

17. Nitsche MA, Paulus W. Excitability changes induced in the human motor cortex by weak transcranial direct current stimulation. J Physiol. (2000) 527:633-9. doi: 10.1111/j.1469-7793.2000.t01-1-00633.x

18. Stagg CJ, Nitsche MA. Physiological basis of transcranial direct current stimulation. Neuroscoentist. (2011) 17:37-53. doi: 10.1177/1073858410386614

19. Nitsche MA, Cohen LG, Wassermann EM, Priori A, Lang N, Antal A, et al. Transcranial direct current stimulation: state of the art 2008. Brain Stimul. (2008) 1:206-23. doi: 10.1016/j.brs.2008.06.004

20. Grefkes C, Fink GR. Recovery from stroke: current concepts and future perspectives. Neurol Res Pract. (2020). 2:17. doi: 10.1186/s42466-020-00060-6

21. Bolognini N, Vallar G, Casati C, Latif LA, El-Nazer R, Williams J, et al. Neurophysiological and behavioral effects of tDCS combined with constraintinduced movement therapy in poststroke patients. Neurorehabil Neural Repair. (2011) 25:819-29. doi: 10.1177/1545968311411056

22. Lindenberg R, Renga V, Zhu LL, Nair D, Schlaug G. Bihemispheric brain stimulation facilitates motor recovery in chronic stroke patients. Neurology. (2010) 75:2176-84. doi: 10.1212/WNL.0b013e318202013a

23. Lindenberg R, Zhu LL, Schlaug G. Combined central and peripheral stimulation to facilitate motor recovery after stroke: the effect of number of sessions on outcome. Neurorehabil Neural Repair. (2012) 26:47983. doi: $10.1177 / 1545968311427568$

24. Nair D, Renga V, Hamelin S, Pascual-Leone A, Schlaug G. Improving motor function in chronic stroke patients using simultaneous occupational therapy and TDCS. Stroke. (2008) 39:542.

25. O'Shea J, Boudrias MH, Stagg CJ, Bachtiar V, Kischka U, Blicher JU, et al. Predicting behavioural response to TDCS in chronic motor stroke. Neuroimage. (2014) 85:924-33. doi: 10.1016/j.neuroimage.2013.05.096

26. Fusco A, Iosa M, Venturiero V, De Angelis D, Morone G, Maglione L, et al. After vs. priming effects of anodal transcranial direct current stimulation on upper extremity motor recovery in patients with subacute stroke. Restor Neurol Neurosci. (2014) 32:301-12. doi: 10.3233/RNN-130349

27. Hummel FC, Voller B, Celnik P, Floel A, Giraux P, Gerloff C, et al. Effects of brain polarization on reaction times and pinch force in chronic stroke. BMC Neurosci. (2006) 7:73. doi: 10.1186/1471-2202-7-73

28. Kim YJ, Ku J, Cho S, Kim HJ, Cho YK, Lim T, et al. Facilitation of corticospinal excitability by virtual reality exercise following anodal transcranial direct current stimulation in healthy volunteers and subacute stroke subjects. $J$ Neuroeng Rehabil. (2014) 11:124. doi: 10.1186/1743-0003-11-124

29. Stagg CJ, Bachtiar V, O’Shea J, Allman C, Bosnell RA, Kischka U, et al. Cortical activation changes underlying stimulation-induced behavioural gains in chronic stroke. Brain. (2012) 135:276-84. doi: 10.1093/brain/ awr313

30. Boggio PS, Nunes A, Rigonatti SP, Nitsche MA, Pascual-Leone A, Fregni F. Repeated sessions of noninvasive brain DC stimulation is associated with motor function improvement in stroke patients. Restor Neurol Neurosci. (2007) 25:123-9.

31. Kim DY, Lim JY, Kang EK, You DS, Oh MK, Oh BM, et al. Effect of transcranial direct current stimulation on motor recovery in patients with subacute stroke. Am J Phys Med Rehabil. (2010) 89:879-86. doi: 10.1097/PHM.0b013e3181f70aa7

32. Zimerman M, Heise KF, Hoppe J, Cohen LG, Gerloff C, Hummel FC. Modulation of training by single-session transcranial direct current stimulation to the intact motor cortex enhances motor skill acquisition of the paretic hand. Stroke. (2012) 43:218591. doi: 10.1161/STROKEAHA.111.645382.Modulation

33. Straudi S, Fregni F, Martinuzzi C, Pavarelli C, Salvioli S, Basaglia $\mathrm{N}$. tDCS and robotics on upper limb stroke rehabilitation: effect modification by stroke duration and type of stroke. Biomed Res Int. (2016) 2016:5068127. doi: 10.1155/2016/5068127

34. Hesse S, Waldner A, Mehrholz J, Tomelleri C, Pohl M, Werner C. Combined transcranial direct current stimulation and robotassisted arm training in subacute stroke patients: an exploratory, randomized multicenter trial. Neurorehabil Neural Repair. (2011) 25:838-46. doi: 10.1177/1545968311413906

35. Khedr EM, Shawky OA, El-Hammady DH, Rothwell JC, Darwish ES, Mostafa OM, et al. Effect of anodal versus cathodal transcranial direct current stimulation on stroke rehabilitation: a pilot randomized controlled trial. Neurorehabil Neural Repair. (2013) 27:592-601. doi: $10.1177 / 1545968313484808$

36. Lee SJ, Chun MH. Combination transcranial direct current stimulation and virtual reality therapy for upper extremity training in patients with subacute stroke. Arch Phys Med Rehabil. (2014) 95:431-8. doi: 10.1016/j.apmr.2013.10.027

37. Nair DG, Renga V, Lindenberg R, Zhu L, Schlaug G. Optimizing recovery potential through simultaneous occupational therapy and non-invasive brain-stimulation using tDCS. Restor Neurol Neurosci. (2011) 29:41120. doi: 10.3233/RNN-2011-0612.Optimizing

38. Pavlova EL, Lindberg P, Khan A, Ruschokowski S, Nitsche MA, Borg J. Transcranial direct current stimulation combined with visuo-motor training as treatment for chronic stroke patients. Restor Neurol Neurosci. (2017) 35:307-17. doi: 10.3233/RNN-160706

39. Lindberg PG, Roche N, Robertson J, Roby-Brami A, Bussel B, Maier MA. Affected and unaffected quantitative aspects of grip force control in hemiparetic patients after stroke. Brain Res. (2012) 1452:96-107. doi: 10.1016/j.brainres.2012.03.007

40. Kurillo G, Gregori,č M, Goljar N, Bajd T. Grip force tracking system for assessment and rehabilitation of hand function. Technol Health Care. (2005) 13:137-49. doi: 10.3233/thc-2005-13301

41. Seo NJ, Rymer WZ, Kamper DG. Delays in grip initiation and termination in persons with stroke: effects of arm support and active muscle stretch exercise. J Neurophysiol. (2009) 101:3108-15. doi: 10.1152/jn.91108.2008

42. Harris JE, Eng JJ. Strength training improves upper-limb function in individuals with stroke: a meta-analysis. Stroke. (2010) 41:136-40. doi: 10.1161/STROKEAHA.109.567438

43. Bradnam LV, Stinear CM, Barber PA, Byblow WD. Contralesional hemisphere control of the proximal paretic upper limb following stroke. Cereb Cortex. (2012) 22:2662-71. doi: 10.1093/cercor/bhr344 
44. Fugl-Meyer A, Jääskö L, Leyman I, Olsson S, Steglind S. The post-stroke hemiplegic patien. Scand J Rehabil Med. (1975) 7:13-31.

45. Wolf SL, Winstein CJ, Miller JP, Taub E, Uswatte G, Morris D, et al. Effect of constraint-induced movement. JAMA. (2006) 296:2095104. doi: 10.1001/jama.296.17.2095

46. Zeller D, Aufm Kampe K, Biller A, Stefan K, Gentner R, Schütz A, et al. Rapid-onset central motor plasticity in multiple sclerosis. Neurology. (2010) 74:728-35. doi: 10.1212/WNL.0b013e3181d31dcf

47. Gandiga PC, Hummel FC, Cohen LG. Transcranial DC stimulation (tDCS): a tool for double-blind sham-controlled clinical studies in brain stimulation. Clin Neurophysiol. (2006) 117:845-50. doi: 10.1016/j.clinph.2005.12.003

48. Bikson M, Grossman P, Thomas C, Zannou AL, Jiang J, Adnan T, et al. Safety of transcranial direct current stimulation: evidence based update 2016. Brain Stimul. (2016) 9:641-61. doi: 10.1016/j.brs.2016.06.004

49. Bates D, Maechler M, Bolker B, Walker S. Fitting linear mixed-effects models using lme4. J Stat Softw. (2015) 67:1-48. doi: 10.18637/jss.v067.i01

50. Jaeger B. r2glmm: Computes $R$ Squared for Mixed (Multilevel) Models. R Package Version 0.1.2. (2017). Available online at: https://CRAN.R-project. org/package $=$ r2glmm (accessed April 14, 2020).

51. Lüdecke D. ggeffects: tidy data frames of marginal effects from regression models. J Open Sour Softw. (2018) 3:772. doi: 10.21105/joss.00772

52. R Core Team. R: A Language and Environment for Statistical Computing. Vienna: R Foundation for Statistical Computing (2020).

53. Wickham H, Averick M, Bryan J, Chang W, McGowan L, François $\mathrm{R}$, et al. Welcome to the tidyverse. J Open Sour Softw. (2019) 4:1686. doi: 10.21105/joss.01686
54. Verbeke G, Molenberghs G. Linear Mixed Models for Longitudinal Data. Springer Series in Statistics. New York, NY: Springer (2000).

55. Hamoudi M, Schambra HM, Fritsch B, Schoechlin-Marx A, Weiller C, Cohen LG, et al. Transcranial direct current stimulation enhances motor skill learning but not generalization in chronic stroke. Neurorehabil Neural Repair. (2018) 32:295-308. doi: 10.1177/1545968318769164. Transcranial

Conflict of Interest: The authors declare that the research was conducted in the absence of any commercial or financial relationships that could be construed as a potential conflict of interest.

Publisher's Note: All claims expressed in this article are solely those of the authors and do not necessarily represent those of their affiliated organizations, or those of the publisher, the editors and the reviewers. Any product that may be evaluated in this article, or claim that may be made by its manufacturer, is not guaranteed or endorsed by the publisher.

Copyright (c) 2021 Taud, Lindenberg, Darkow, Wevers, Höflin, Grittner, Meinzer and Flöel. This is an open-access article distributed under the terms of the Creative Commons Attribution License (CC BY). The use, distribution or reproduction in other forums is permitted, provided the original author(s) and the copyright owner(s) are credited and that the original publication in this journal is cited, in accordance with accepted academic practice. No use, distribution or reproduction is permitted which does not comply with these terms. 